\title{
Modeling and Implementation of a Self-Reference Voltage Pre-Regulator
}

\author{
Feng Wei, Dai Yujie, Zhang Xiaoxing, Lv Yingjie \\ College of Electronic Information and Optical Engineering, Nankai University, \\ Tianjin 300071, China \\ fengwei720720@163.com
}

\begin{abstract}
The paper proposes a novel self-reference voltage pre-regulator for LED driver chip. The system modeling of the self-reference voltage pre-regulator is established, which is composed of pre-regulator circuit and reference voltage generating circuit. The reference voltage generating circuit generates a reference voltage, in which the circuit returns the reference voltage as the reference voltage of pre-regulator circuit, therefore, the preregulator outputs a stable voltage for the LED driver chip. In this paper, the circuit implementation is described in detailed, in order to enhance the stability of the system, the miller compensation is used to the circuit, which makes the system more stable. Simulations of the circuit show that the pre-regulator not only can output a stable voltage for the chip, but also the load regulation, line regulation and power support ripple rejection of the pre-regulator exhibit high performance.
\end{abstract}

Keywords: The Self-Reference Voltage Pre-Regulator, System Modeling, Circuit Implementation, Stable Output Voltage

\section{Introduction}

As the progress of light emitting diode (LED) technology, the LED lighting is becoming as a promising illumination solution and a more and more popular choice consider in general[1-4]. There are many advantages of LED lighting, such as high efficiency, longer life time, fast response, low power, smaller size and less pollution, these make LED lighting is widely used in residential lighting, traffic lighting, flash lighting and so on[5-9].

However, the LED driver chip has a power supply question. AC line is rectified, and is connected to an output capacitor of chip. Therefore, an instantaneous input and output power difference exists in a half line cycle when the charge and discharge action in every half line cycle, as shown in [10], this makes the power supply of LED driver chip is unstable. Traditional design uses a zener diode in the chip, which provides power for the chip. However, if there is not zener diode in the process, or the process has zener diode, but the voltage of zener diode is not needed for the chip, therefore, using zener diode is not suitable.

In order to provide a stable voltage for the LED driver chip, a novel self-reference voltage pre-regulator is designed. Using the feedback mechanism, the pre-regulator not only can output stable voltage for the LED driver chip, but also the reference voltage is generated by it-self. 


\section{Modeling of the Pre-Regulator}

\subsection{System Modeling of the Pre-Regulator}

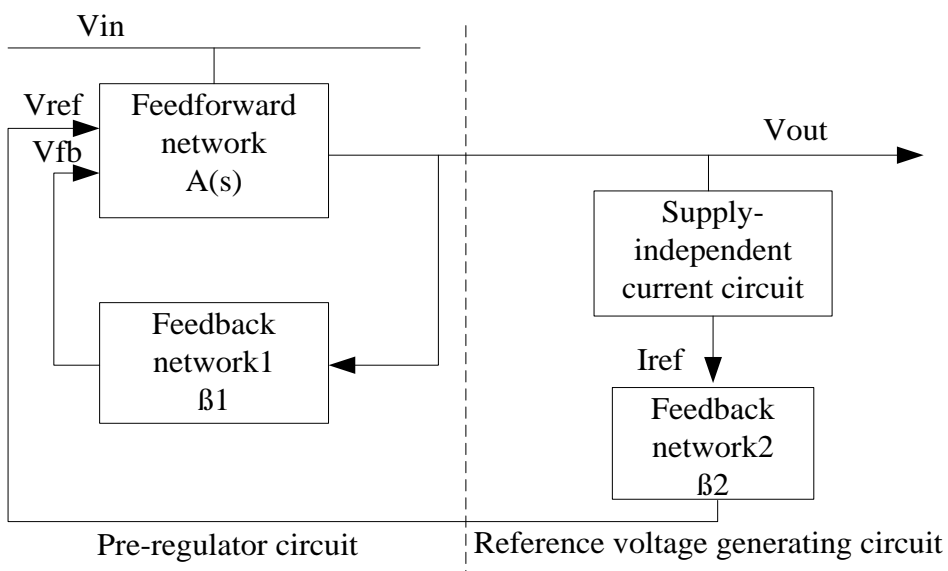

\section{Figure 1: System Modeling of the Pre-Regulator}

The system modeling of pre-regulator is shown in Figure 1 . The voltage $V_{\text {in }}$ is power supply, which comes from outside of the chip. Pre-regulator is composed of the pre-regulator circuit and reference voltage generating circuit. Pre-regulator circuit outputs a stable output voltage $V_{\text {out }}$, which is used to power for the other blocks of chip and is returned by feedback network1 as the feed-forward network input voltage $V_{f b}$. Reference voltage generating circuit is powered by the output voltage $V_{\text {out }}$ and outputs reference current $I_{\text {ref }}$, which is independent of the output voltage $V_{\text {out }}$. The feedback network 2 senses the reference current $I_{r e f}$ and returns a reference voltage $V_{\text {ref }}$, which can be written as:

$$
V_{r e f}=\beta_{2} I_{r e f}
$$

Where, $\beta_{2}$ is feedback factor of the feedback network2, because the reference current $I_{r e f}$ is independent of the output voltage $V_{\text {out }}$, so the reference voltage $V_{\text {ref }}$ is also independent of the output voltage $V_{\text {out }}$.

As shown in figure 1 , the output voltage $V_{\text {out }}$ can be expressed by the following equation:

$$
V_{\text {out }}=\frac{A_{o p}(s)}{1+A_{o p}(s) \beta_{1}} \operatorname{Vref}
$$

Where, $A_{o p}(s)$ is the open loop transfer function of the feed-forward network, $\beta_{1}$ is feedback factor of the feedback network1. The equations of (1) and (2) exhibit that output voltage $V_{\text {out }}$ is independent of the power supply $V_{\text {in }}$.

\subsection{Structure Modeling of the Pre-regulator}

Figure 2 shows a structure modeling of the pre-regulator. Feed-forward network is composed of error amplifier, buffer and power output stage. Error amplifier senses and amplifies the feedback errors, so the accuracy is vital parameter in the 
design of error amplifier, and error amplifier must respond quickly to restrain the errors of output voltage $V_{\text {out }}$ which are caused by the rapid load changes and power supply ripple.

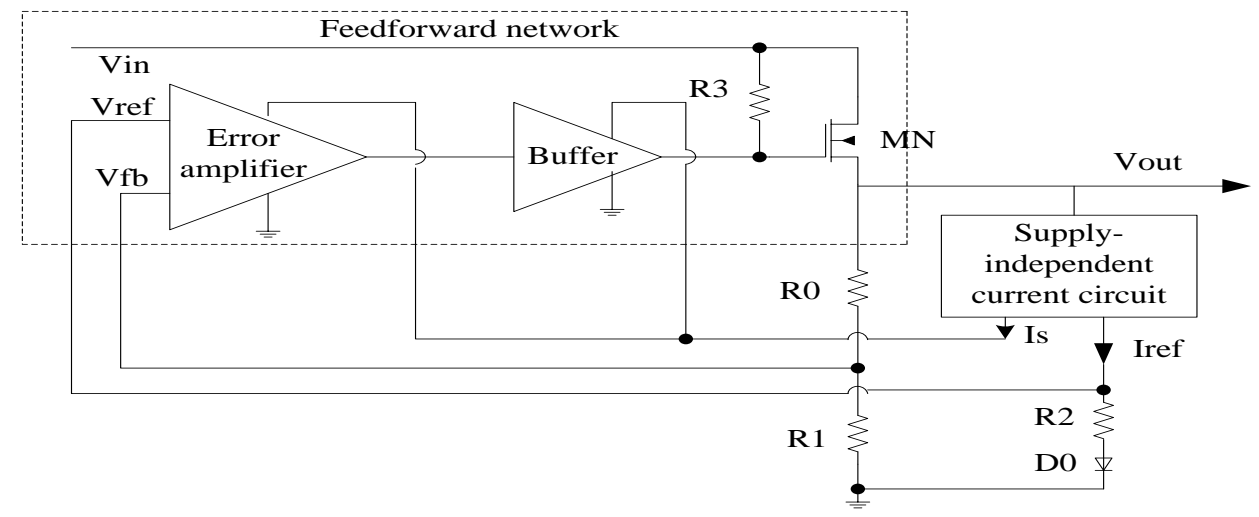

Figure 2: Structure Modeling of the Pre-Regulator

The buffer is necessary for the pre-regulator circuit. Because the output voltage of error amplifier is powered by supply-independent current circuit as shown in Figure ure 2, so the power supply of error amplifier is low, which causes the output voltage of error amplifier is low, but the power output switch $\mathrm{MN}$ is a n-type MOSFET, which gate requires high voltage to drive, therefore, buffer shifts the output voltage of error amplifier and the input voltage of the power output stage to make the circuit work better. And there are large parasitic capacitances in the power output switch MN, in order to make power output switch response quickly, buffer is necessary, because buffer can provide sufficient current and voltage for the power output switch.

The power output stage can provide stable voltage for load. Using the n-type MOSFET as the power output switch is that power output stage is a source follower, which exhibits a small impedance approximately the reciprocal of the transconductance of the power output switch MN, this means that power output stage can respond quickly when load changed rapidly, therefore, the n-type is selected.

The feedback network 1 is a resistive divider as shown in Figure ure 2, which is a frequency-independent quantity. The feedback factor can be calculated as following equation:

$$
\beta_{1}=R_{1} /\left(R_{0}+R_{1}\right)
$$

Therefore, the feedback voltage $V_{f b}$ can be expressed by the following equation:

$$
V_{f b}=\beta_{1} V_{\text {out }}
$$

Hence, a fraction of the output voltage $V_{\text {out }}$ is sensed by feedback network1 and compared with the input of error amplifier, generated an error term. Thus, in a welldesigned feedback system, the error term is minimized.

As shown in Figure 2, supply-independent current circuit outputs current $I_{s}$ and $I_{r e f}$, which are independent of the output voltage $V_{\text {out }}$. The current $I_{s}$ provides power supply for error amplifier and buffer, current $I_{\text {ref }}$ flows through the resistor $R_{2}$ and diode $D_{0}$ to generate the reference voltage $V_{r e f}$, therefore, the $V_{\text {ref }}$ can be calculated as: 


$$
V_{\text {ref }}=\beta_{2} I_{\text {ref }}=R_{2} I_{\text {ref }}+V_{\text {diode }}
$$

Where, voltage $V_{\text {diode }}$ is threshold voltage of diode $D_{0}$.

\section{Circuit Implementation}

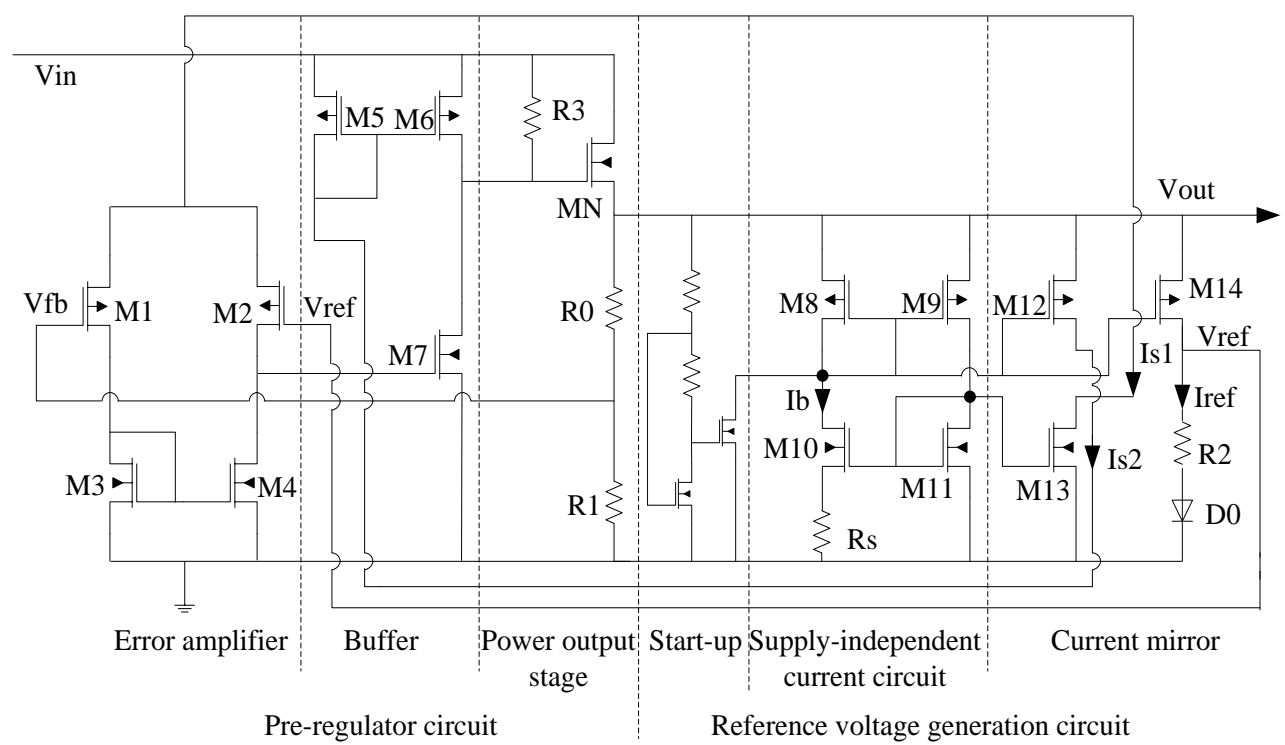

Figure 3: Circuit Implementation

Error amplifier is a double-end input single-end output differential amplifier, which is composed of MOSFET $M_{1} \sim M_{4}$. The input signals of error amplifier are voltage $V_{r e f}$ and $V_{f b}$, the output signal of error amplifier is connected to buffer, power supply is the current $I_{s 1}$ of reference voltage generation circuit as shown in figure 3 . The low-frequency gain $A_{(E A)}(0)$ of the error amplifier can be expressed as following equation:

$$
A_{(E A)}(0)=g_{m(M 1)} R_{\text {out }(E A)}
$$

Where, $g_{m(M 1)}$ is the transconductance of M1, $R_{\text {out }(E A)}$ is the output resistance of the error amplifier, $R_{\text {out }(E A)}$ can be calculated as:

$$
R_{\text {out }(E A)}=r_{o(M 2)} / / r_{o(M 4)}
$$

Where, $r_{O(M 2)}$ is the output resistance of M2, $r_{o(M 4)}$ is the output resistance of M4.

Buffer is a common-source operational amplifier which is composed of MOSFET M5, M6 and M7. M7 converts an error amplifier output change $\Delta V_{\text {out (EA) }}$ to a drain current change $g_{m(M 7)} \Delta V_{\text {out }(E A)}$, hence the buffer has an output voltage change $g_{m(M 7)} R_{\text {out (buffer })} \Delta V_{\text {out }(E A)}$, so the low-frequency gain $A_{(b u f f e r)}(0)$ of the buffer is expressed as following equation:

$$
A_{(\text {buffer })}(0)=-g_{m(M 7)} R_{\text {out }(\text { buffer })}
$$


Where, $g_{m(M 7)}$ is the transconductance of M7, $R_{\text {out }(\text { buffer })}$ is the output resistance of the buffer, $R_{\text {out(buffer) }}$ can be calculated as following equation:

$$
R_{\text {out }(\text { buffer })}=r_{o(M 6)} / / \mathrm{ro}_{(M 7)} / / R_{3}
$$

Where, $r_{O(M 6)}$ is the output resistance of $\mathrm{M} 6, r o_{(M 7)}$ is the output resistance of $\mathrm{M} 7, R_{3}$ is resistor, which can provide a bias voltage for power output switch MN.

The power output stage is source follower, so the low-frequency gain $A_{(\text {power_output })}(0)$ of output power state is approximate $1 . R_{\text {out }}$ is the output resistance of power output stage, which can be calculated as:

$$
R_{\text {out }}=\left(R_{0}+R_{1}\right) / /\left(1 / g_{m(M N)}\right) \approx 1 / g_{m(M N)}
$$

$g_{m(M N)}$ is the transconductance of power output switch MN.

Thus, the low-frequency open loop gain $A_{o p}(0)$ of pre-regulator circuit can be expressed as following equation:

$$
\begin{aligned}
& A_{\text {op }}(0)=A_{(E A)}(0) A_{(\text {buffer })}(0) A_{(\text {power_output })}(0) \\
& =-\left(g_{m(M 1)} R_{\text {out }(E A)} g_{m(M 7)} R_{\text {out }(\text { buffer })}\right)
\end{aligned}
$$

From equation (2), the low-frequency power output voltage $V_{\text {out }}$ can be obtained:

$$
V_{\text {out }}=\frac{A_{o p}(0)}{1+A_{o p}(0) \beta_{1}} \operatorname{Vref}=\left[\frac{1}{\beta_{1}}\left(1-\frac{1}{1+A_{o p}(0) \beta_{1}}\right)\right] \text { Vref }
$$

Equation (12) implies that increasing the value of the low-frequency gain $A_{o p}(0) \beta_{1}$, the error of the low-frequency power output voltage $V_{\text {out }}$ can be reduced.

The supply-independent current circuit generates current $I_{b}$ as depicted in figure 3 . The current $I_{b}$ can be calculated as:

$$
I_{b}=\frac{2}{\mu_{n} C_{o x}(W / L)_{M 11}} \frac{1}{R_{s}^{2}}\left(1-\frac{1}{\sqrt{K_{1}}}\right)^{2}
$$

Where, $\mu_{n} C_{o x}$ is the device transconductance parameter of the n-type MOSFET, $(W / L)_{M 10}$ and $(W / L)_{M 11}$ are the width to length ratio of M10 and M11, $K_{1}$ is given as:

$$
K_{1}=\frac{(W / L)_{(M 10)}}{(W / L)_{(M 11)}}
$$

Equations (13) and (14) imply that the current $I_{b}$ is independent of the output voltage $V_{\text {out }}$. Reference current $I_{\text {ref }}$ can be calculated as:

$$
I_{\text {ref }}=K_{2} I_{b}
$$

Where, $K_{2}$ is written as:

$$
K_{2}=\frac{(W / L)_{(M 14)}}{(W / L)_{(M 8)}}
$$


Thus, the output voltage $V_{\text {out }}$ can be obtained from equations (2), (5), (13) and (15),

$$
V_{\text {out }}=\frac{A(s)}{1+A(s) \beta_{1}} \beta_{2} K_{2} \frac{2}{\mu_{n} C_{o x}(W / L)_{M 11}} \frac{1}{R_{s}^{2}}\left(1-\frac{1}{\sqrt{K_{1}}}\right)^{2}
$$

\section{Stability Analysis and Frequency Compensation}

Because the reference voltage $V_{\text {ref }}$ is independent of the out voltage $V_{\text {out }}$, the reference voltage $V_{\text {ref }}$ does not affect the stability of the circuit. Therefore, that only analyses the pre-regulator circuit, which is a negative feedback network. Figure ure 4 (a) shows the structure modeling of the pre-regulator circuit, and Figure ure 4 (b) shows the small-signal equivalent circuit of the pre-regulator circuit. As shown in figure 4 (b), where, $G_{(E A)}$ is the transconductance of the error amplifier, $R_{\text {out }(E A)}$ is the output resistance of the error amplifier, $C_{P(E A)}$ is the output terminal parasitic capacitance of the error amplifier. $G_{(b u f f e r)}$ is the transconductance of the buffer, $R_{\text {out(buffer) }}$ is the output resistance of the error buffer, $C_{P(b u f f e r)}$ is the output terminal parasitic capacitance of the buffer, $G_{(\text {switch }}$ is the transconductance of the power output stage, $R_{\text {out }}$ is the output resistance of the power output stage, $C_{P(\text { out })}$ is the output terminal parasitic capacitance of power output stage.

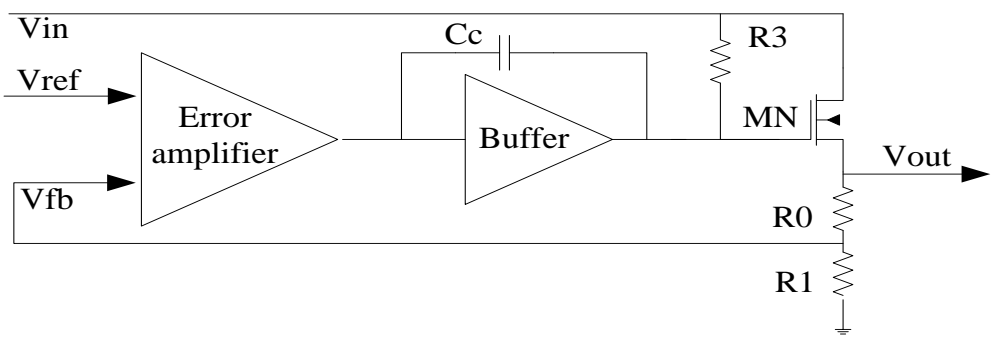

(a) The Structure Modeling Of Pre-Regulator Circuit

(b)

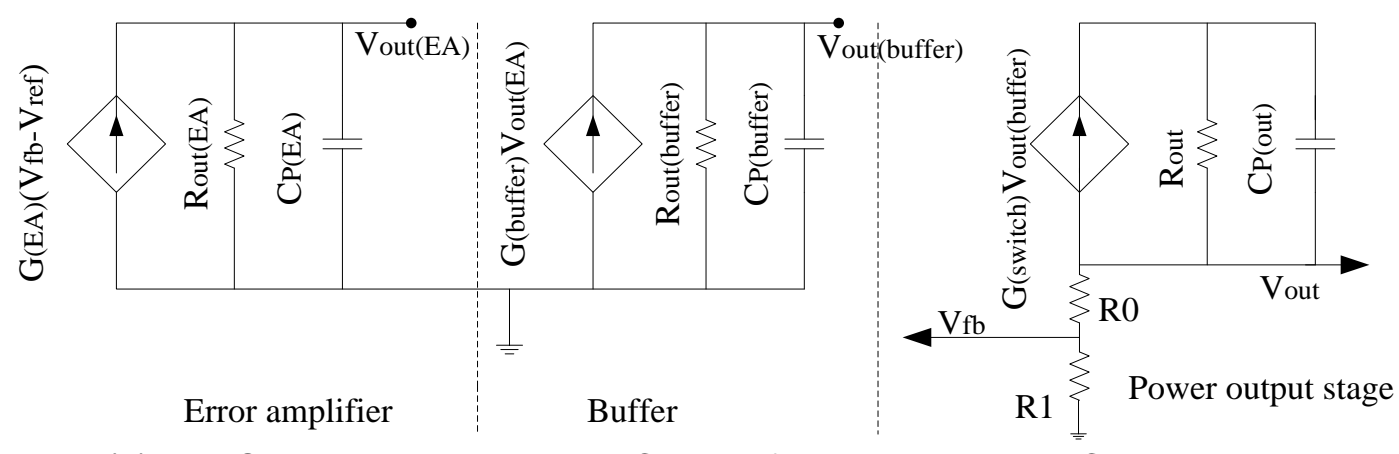

(b) The Small-signal Equivalent Circuit of the Pre-regulator Circuit.

Figure 4: The Pre-Regulator Circuit Topology

Before compensation, there are three poles in the circuit as shown in figure 4 (b), which can be calculated as: 


$$
\begin{gathered}
P_{\text {out }(E A)}=-\frac{1}{R_{\text {out }(E A)} C_{P(E A)}}=-\frac{1}{\left(r_{o(M 2)} / / r_{o(M 4)}\right) C_{P(E A)}} \\
P_{\text {out (buffer })}=-\frac{1}{R_{\text {out (buffer })} C_{P(\text { buffer })}}=-\frac{1}{\left(r_{o(M 6)} / / r_{o(M 7)} / / R_{3}\right) C_{P(\text { buffer })}}
\end{gathered}
$$

And

$$
P_{\text {out }}=-\frac{1}{R_{\text {out }} C_{P(\text { out })}}=-\frac{g_{m(M N)}}{C_{P(E A)}}
$$

Where, $P_{\text {out }(E A)}$ is an output terminal pole of error amplifier, $P_{\text {out(buffer })}$ is an output terminal pole of buffer, $P_{\text {out }}$ is an output terminal pole of power output stage. The value of the $R_{\text {out (EA) }}, R_{\text {out (buffer) }}$ and $R_{\text {out }}$ can be obtained from equations (7), (9) and (10). From the equations (18), (19) and (20), the three poles relation can be expressed as:

$$
P_{\text {out }(E A)}, P_{\text {out }(\text { buffer })} \leq P_{\text {out }}
$$

Therefore, the output terminal pole $P_{\text {out }}$ of power output stage is a high-frequency pole, which does not affect the stability of the circuit, so the pole $P_{\text {out }}$ can be ignored.

Because the relative position of $P_{\text {out }(E A)}$ and $P_{\text {out (buffer })}$ is relatively close, hence, the circuit exhibits two dominant poles, so the phase approaches $-180^{\circ}$. In other words, the phase margin of the circuit maybe close to zero, this makes the system suffer from instability.

In order to enhance the stability of the system, Miller compensation is used for frequency compensation. The Miller capacitor $C_{c}$ is connected from the output to the input of the buffer as shown in figure 4 (a). After compensation, the pole $P_{\text {out }(E A)(\text { Miller })}$ can be expressed as:

$$
P_{\text {out }(E A)(\text { Miller })}=-\frac{1}{R_{\text {out }(E A)}\left[C_{P(E A)}+\left(1+A_{(\text {buffer })}(0)\right) C_{C}\right]}
$$

Where, $C_{c}$ is the Miller capacitor.

The pole $P_{\text {out }(\text { buffer)(Miller) }}$ can be written as:

$$
P_{\text {out(buffer })(\text { Miller })}=-\frac{g_{m(M 7)}}{C_{P(\text { buffer })}}
$$

Where, $g_{m(M 7)}$ is the transconductance of M7. Comparing $P_{\text {out }(E A)(\text { Miller })}$ with the $P_{\text {out }(E A)}$, Miller compensation moves the $P_{\text {out }(E A)}$ closer to the origin of complex

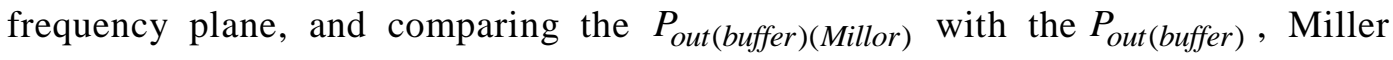
compensation moves away the $P_{\text {out(buffer) }}$ from the origin of the complex frequency plane, the frequency compensation improves the phase margin as shown in figure 5 . 


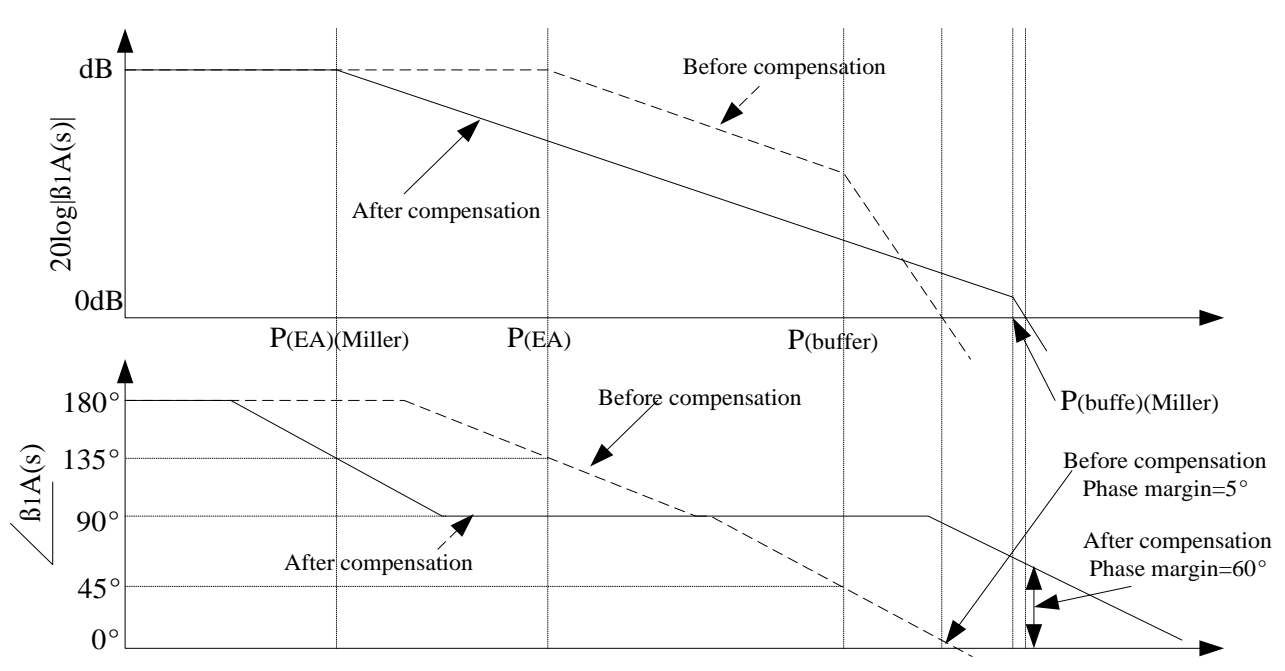

Figure 5: Bode Plots of Before Compensation and After Compensation

\section{Simulation and Analysis}

The circuit is simulated based on BCD350 process. Because the pre-regulator acts in the chip, and the range of input voltage $V_{i n}$ is $8 \sim 30 \mathrm{~V}$, therefore, the output voltage $V_{\text {out }}$ must be stabilized within the range of input voltage $V_{\text {in }}$. As the shown in figure 6 , when the input voltage $V_{i n}$ is $7.2 \mathrm{~V}$, the output voltage is $4.99914 \mathrm{~V}$, and when the input voltage $V_{i n}$ is $30 \mathrm{~V}$, the output voltage is $5.00119 \mathrm{~V}$, there is only a difference of $2.05 \mathrm{mV}$.

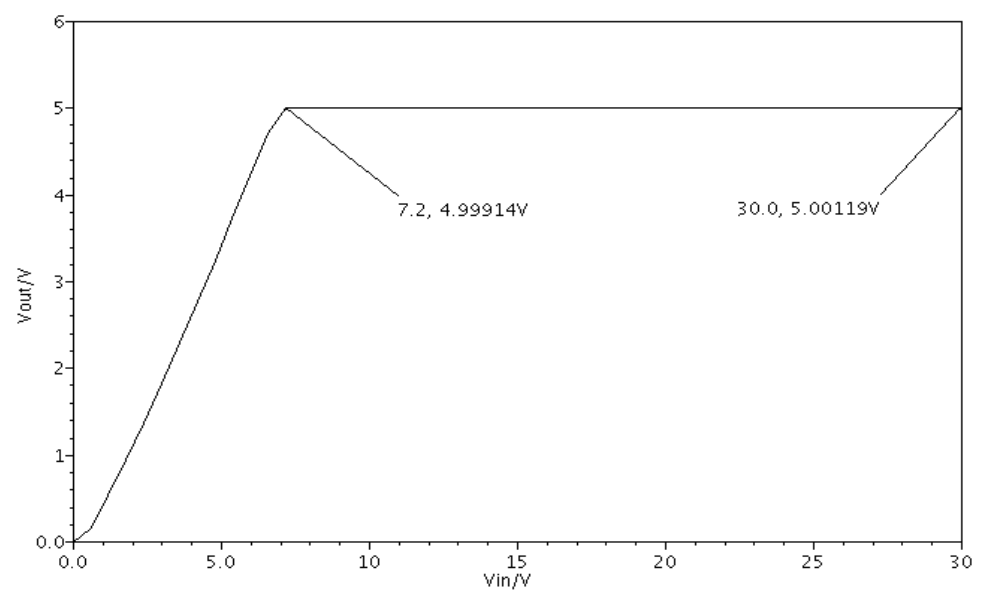

Figure 6: DC of Simulation of Input Voltage

Load regulation (LDR) is an important performance for pre-regulator, which reflects a change of output voltage $V_{\text {out }}$ variation resulting from the load current $I_{\text {load }}$ variation. The LDR can be expressed as following equation:

$$
L D R=\frac{\Delta V_{\text {out }}}{\Delta I_{\text {load }}}
$$

Where, $\Delta V_{\text {out }}$ is variation of the output voltage $V_{\text {out }}, \Delta I_{\text {load }}$ is the variation of the load current $I_{\text {load }}$. Figure ure 7 shows the waveforms of the LDR, when the load current from 
zero to $100 \mathrm{~mA}$, the input voltage is $11 \mathrm{~V}, 12 \mathrm{~V}$ and $13 \mathrm{~V}$. Table 1 shows the LDR of the pre-regulator.

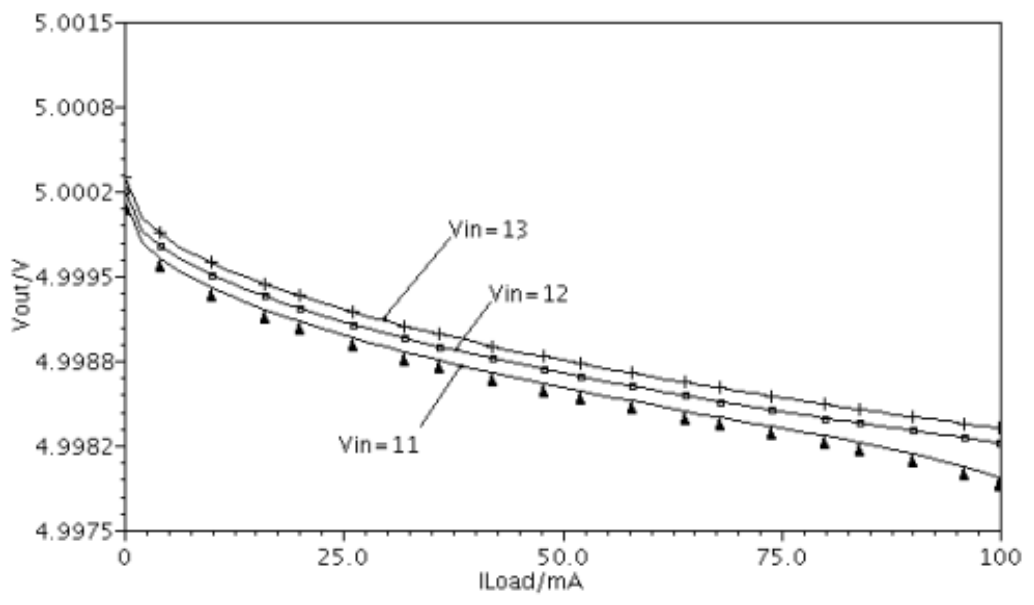

Figure 7: The Waveforms of LDR

Table 1: LDR ,When The Load Current From 0 To 100ma, Input Voltage Is 11V, 12V And 13V

\begin{tabular}{l|l|l}
\hline$V_{\text {in }}$ & $\Delta V_{\text {out }}$ & $L D R$ \\
\hline$V_{\text {in }}=11 \mathrm{~V}$ & $\Delta V_{\text {out }}=5.00008 \mathrm{~V}-4.99791 \mathrm{~V}=2.17 \mathrm{mV}$ & $L D R=2.17 \mathrm{mV} / 100 \mathrm{~mA}$ \\
\hline$V_{\text {in }}=12 \mathrm{~V}$ & $\Delta V_{\text {out }}=5.00018 \mathrm{~V}-4.99819 \mathrm{~V}=1.99 \mathrm{mV}$ & $L D R=1.99 \mathrm{mV} / 100 \mathrm{~mA}$ \\
\hline$V_{\text {in }}=13 \mathrm{~V}$ & $\Delta V_{\text {out }}=5.00027 \mathrm{~V}-4.9983 \mathrm{~V}=1.99 \mathrm{mV}$ & $L D R=1.97 \mathrm{mV} / 100 \mathrm{~mA}$ \\
\hline
\end{tabular}

Line regulation (LNR) is also a vital performance, which describes the output voltage $V_{\text {out }}$ variation arising from change in the input voltage $V_{i n}$, which can be expressed as:

$$
L N R=\frac{\Delta V_{\text {out }}}{\Delta V_{\text {in }}}
$$

Where, $\Delta V_{\text {out }}$ is variation of the output voltage $V_{\text {out }}, \Delta V_{\text {in }}$ is the variation of the input voltage $V_{i n}$. Table 2 shows the LNR, when input voltage $V_{\text {in }}$ from $11 \mathrm{~V}$ to $12 \mathrm{~V}$, load current $I_{\text {load }}$ is $1 \mathrm{~mA}, 10 \mathrm{~mA}$ and $100 \mathrm{~mA}$, the waveforms of LNR are shown in figure 8 . 


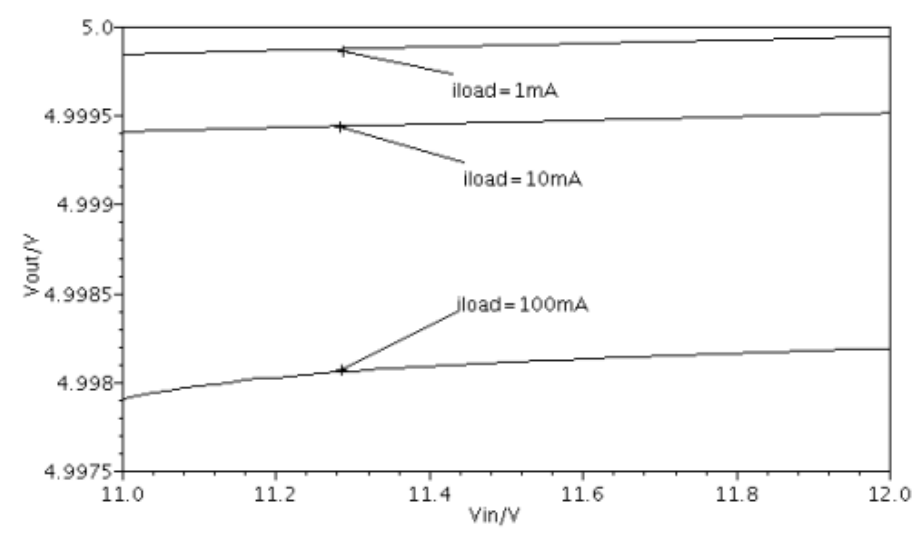

Figure 8: The Waveforms of LNR

Table 2: LNR, When Input Voltage from $11 \mathrm{~V}$ to $12 \mathrm{~V}$, Load Current Is $1 \mathrm{ma}$, $10 \mathrm{ma}$ and $100 \mathrm{ma}$

\begin{tabular}{c|l|l}
\hline$I_{\text {load }}$ & $\Delta V_{\text {out }}$ & $L N R$ \\
\hline$I_{\text {load }}=1 \mathrm{~mA}$ & $\Delta V_{\text {out }}=4.999943 \mathrm{~V}-4.99984 \mathrm{~V}=0.103 \mathrm{mV}$ & $L D R=0.103 \mathrm{mV} / \mathrm{V}$ \\
\hline$I_{\text {load }}=10 \mathrm{~mA}$ & $\Delta V_{\text {out }}=4.999511 \mathrm{~V}-4.99941 \mathrm{~V}=0.101 \mathrm{mV}$ & $L D R=0.101 \mathrm{mV} / \mathrm{V}$ \\
\hline$I_{\text {load }}=100 \mathrm{~mA}$ & $\Delta V_{\text {out }}=4.998188 \mathrm{~V}-4.99791 \mathrm{~V}=0.278 \mathrm{mV}$ & $L D R=0.278 \mathrm{mV} / \mathrm{V}$ \\
\hline
\end{tabular}

Power supply ripple rejection (PSRR) refers broadly to the ability of the circuit to regulate its output voltage $V_{\text {out }}$ against small-signal variation in the input voltage $V_{i n}$. The figure 9 shows the waveform of PSRR. As the shown in figure 9, when the frequency is $10 \mathrm{KHz}$, the PSRR is $-58 \mathrm{~dB}$.

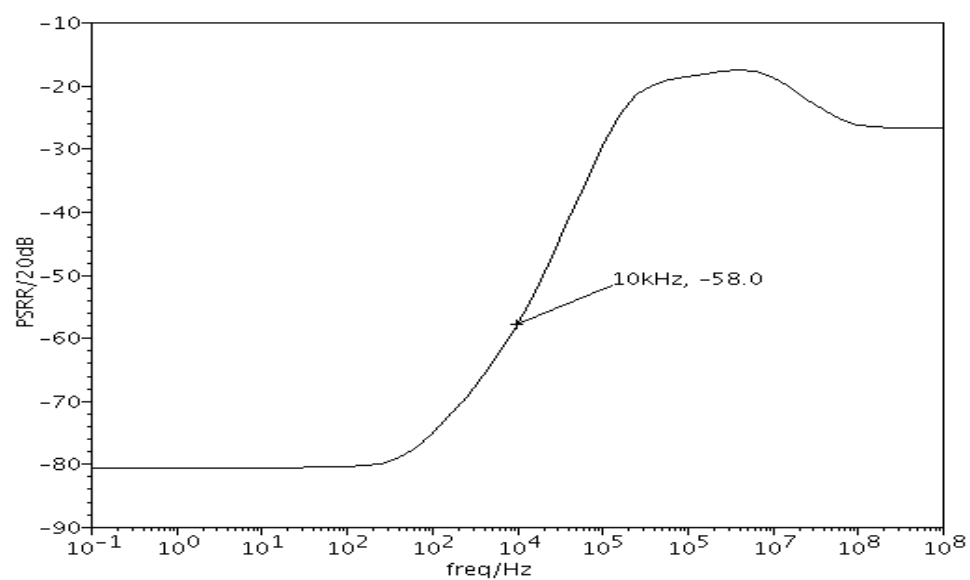

Figure 9: The Waveform of PSRR

\section{Conclusion}

In this paper, a self-reference voltage pre-regulator is proposed for LED driver chip. In order to provide stable voltage for LED driver chip, the pre-regulator circuit and reference 
voltage generating circuit are designed. Using feedback mechanism, the reference voltage is generated by the reference voltage generating circuit and is returned as a reference of the pre-regulator circuit, the pre-regulator circuit outputs a stable voltage for the LED driver chip.

The system modeling and structure modeling is established for the pre-regulator, circuit implementation is described in detail. In order to enhance the stability of system, the Miller compensation is used for frequency compensation.

The results of simulation show that the load regulation, line regulation and power supply ripple rejection are better.

\section{Acknowledgments}

This work was supported by the Tianjin Science and Technology Project (13ZCZDGX04100).

\section{References}

[1] I. S. Eo and G. Ju, "Study on the Effects of Learning by Changing the Color-Temperature LED Lamp", Journal of International Journal of Multimedia and Ubiquitous Engineering, Vol. 9, No.3 (2014).

[2] Y. T. Hsieh, J. Fu, C. L. Fang, H. H. Tsai and Y. Z. Juang, "Design of fast stabilized LED Driver IC with low overcurrent", Proceedings of the IEEE 10th International Conference on Power Electronics and Drive Systems (PEDS), (2013), April, 550-553, Kitakyushu.

[3] J. P. Qiu and L. N. He, "A IED driver IC with constant current and temperature compensation", Proceedings of the 3er IEEE International Conference on Computer Science and Information Technology (ICCSIT), (2010), July, 9-11, Chengdu.

[4] K. I. Hwu, W. C. Tu, and Y. T. Fang, "Dimmable AC LED Driver With Efficiency Improved Based on Switched LED Module", Journal of Display Technology, Vol.10, No.3 (2014).

[5] F. H. Zhang, J. J Ni and Y. J. Yu, "High Power Factor AC-DC LED Driver with Film Capacitors", Journal of Power Electronics, Vol.28, No.10 (2013).

[6] S. Wang, X. B. Ruan, K. Yao, S. Chong, Y. Yang and Z. H. Ye, "A Flicker-Free Electrolytic CapacitorLess AC-DC LED Drive", Journal of Power Electronics, Vol.27, No.11(2012).

[7] X. Xiao, P. Mei, Y. J. Cai and J. X. Wu, "An optocouplerless two-stage high power factor LED driver", Proceedings of the Applied Power Electronics Conference and Exposition (APEC), (2011), March, 20782083, Fort Worth, TX.

[8] H. J. Kim, B. H Lee and C. T.Rim, "Passive LED driver compatible with rapid-start ballast", Proceedings of the 2011 IEEE 8th International Conference on Power Electronics and ECCE Asia (ICPE\&ECCE), (2011), June, 507-514, Jeju.

[9] P. Athalye, M. Harris and G. Negley, "A two-stage LED driver for high-performance high-voltage LED fixtures", Proceedings of the Applied Power Electronics Conference and Exposition (APEC), (2012), February, 2385-2391, Orlando, Fl.

[10] S. Y. Cha, D. H. Park, Yuenjoong Lee, Changseck Lee, Joongho Choi, Jaeshin Lee and Hyobum Lee, "AC-DC converter free LED driver for lightings", Proceedings of the Consumer Electronics (ICCE), (2012), January, 13-16, Las Vegas, NV.

\section{Authors}

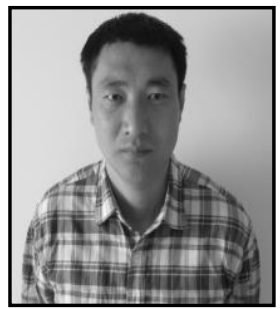

Feng Wei received his Engineer Master degree from Henan University of Science and Technology, China, in 2005. Currently, he is working toward the Ph.D. degree in Nankai University. The main research directions: analog integrated circuit design. 


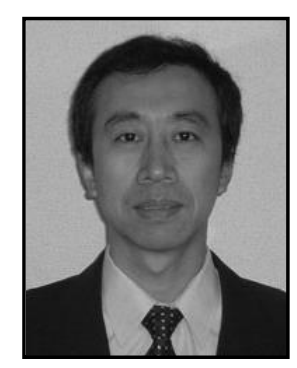

Dai Yujie received his Engineer Master degree from Taiyuan University of Technology, China, in 1984. He received his Ph.D. from TOHOKU UNIVERSITY, Japan, in 1992. The main research directions: the SoC design of nanometer CMOS process, mixed analog-digital integrated circuit, analog integrated circuit.

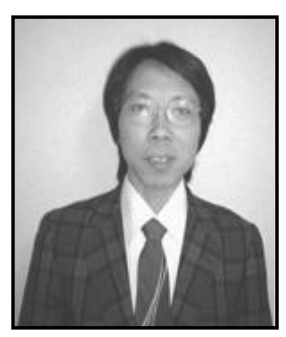

Zhang Xiaoxing received his Engineer Master degree from Kitami Institute of Technology, Japan, in 1995. He received his Ph.D. from Nagaoka University of Technology, Japan, in 1998. The main research directions: the analog processing, analog integrated electronics, mixed analog-digital integrated circuit.

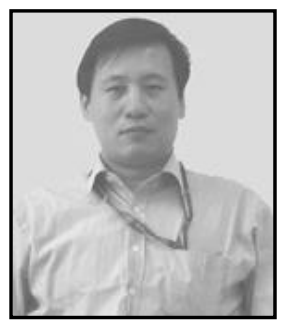

Lv Yingjie received his Engineer Master degree from Hokkaido University, Japan, in 1995, received his second Engineer Master degree in 1997.The main research directions: the digital integrated electronics, mixed analog-digital integrated circuit. 